\title{
A Double-Blind, Randomized, Active Drug Comparative, Parallel-Group, Multi-Center Clinical Study to Evaluate the Safety and Efficacy of Probiotics (Bacillus licheniformis, Zhengchangsheng ${ }^{\circledR}$ capsule) in Patients with Diarrhea
}

\author{
Jun Heo, Sung Kook Kim, Kyung Sik Park ${ }^{1}$, Hye Kyung Jung ${ }^{2}$, Joong Goo Kwon ${ }^{3}$, Byung Ik Jang ${ }^{4}$ \\ Department of Internal Medicine, Kyungpook National University School of Medicine, Daegu, Department of Internal Medicine Division of \\ Gastroenterology, Keimyung University School of Medicine', Daegu, Department of Internal Medicine, Ewha Womans University School of \\ Medicine, Seoul, Department of Internal Medicine, Catholic University of Daegu School of Medicine, Daegu, Department of Internal Medicine, \\ Yeungnam University, College of Medicine ${ }^{4}$, Daegu, Korea
}

Background/Aims: Bacillus Licheniformis, a probiotic used in the treatment of diarrhea, has been shown to suppress the growth of pathologic bacteria. This study was performed to assess the therapeutic efficacy and safety of Zhengchangsheng ${ }^{\circledR}$ (Bacillus Licheniformis) in comparison with another probiotic, Bioflor ${ }^{\circledR}$ (Saccharomyces Boulardii) for the treatment of diarrhea. Methods: Patients with diarrhea $(n=158)$ were randomized to receive Zhengchangsheng ${ }^{\circledR}$ or Bioflor ${ }^{\circledR}$ for 5 days. The existence or non-existence of formed feces, changes in daily stool frequency, improvement of subjective symptoms, and changes in the severity of diarrhea were compared. Results: Of the 158 full analysis set (FAS) patient population, 151 patients comprised the per protocol (PP) analysis. The rates of recovered to formed feces in the Bacillus and Saccharomyces groups were $91.0 \%$ vs. $95.0 \%$ in the FAS $(P=0.326)$ and $90.5 \%$ vs. $96.1 \%$ in the PP analysis $(P=0.169)$, respectively. The mean duration of diarrhea changing to formed feces was $3.15 \pm 1.10$ days in the Bacillus group and $3.22 \pm 1.01$ in the Saccharomyces group $(P=0.695$, FAS). The frequency of defecation, subjective symptoms, and degree of severe diarrhea were improved in both groups, however, there were no statistically significant differences between the 2 groups. Analysis of the $95 \%$ confidence intervals for the differences in the rate of recovery to formed feces between the 2 groups met the criteria for non-inferiority of Bacillus compared to Saccharomyces. No significant adverse events were observed during the study period. Conclusions: Zhengchangsheng ${ }^{\circledR}$ is not inferior to Bioflor ${ }^{\circledR}$ in therapeutic efficacy and is a safe and useful therapeutic agent for the treatment of diarrhea. (Intest Res 2014;12:236-244)

Key Words: Probiotics; Diarrhea; Bacillus Licheniformis

Received November 19, 2013. Revised January 8, 2014.

Accepted February 4, 2014.

Correspondence to Sung Kook Kim, Department of Internal Medicine, Kyungpook National University Hospital, 130 Dongdeok-ro, Jung-gu, Daegu 700-721, Korea. Tel: +82-53-200-5517, Fax: +82-53-200-5505, E-mail: skkim@knu.ac.kr

Financial support: This work was supported by funds from the Aju Pharm. Co., Ltd. Conflict of interest: None.

\section{INTRODUCTION}

Diarrhea, characterized by more loose or liquid bowel movements, is often associated with an increase in the number and amount of stools. The World Health Organization (WHO) defines diarrhea as a health disorder involving 3 or more loose or fluid bowel movements per day lasting for more than 2 days. ${ }^{1}$ Among the therapeutic agents for

๑ Copyright 2014. Korean Association for the Study of Intestinal Diseases. All rights reserved.

This is an Open Access article distributed under the terms of the Creative Commons Attribution Non-Commercial License (http://creativecommons.org/licenses/by-nc/3.0)

which permits unrestricted non-commercial use, distribution, and reproduction in any medium, provided the original work is properly cited. 
diarrhea, probiotics have been used for preventing and treating diarrhea through amechanism involving interactions between immune cells and intestinal flora present in the intestinal mucosal barrier. ${ }^{2}$ Probiotics are effective and less invasive and lower in cost than other therapeutic options, with the added benefit of preventing the destruction of healthy intestinal flora. The results of several clinical trials have proven that probiotics were effective in the management and prevention of infectious enteritis, antibiotic-related diarrhea, travel-related diarrhea, and IBS. ${ }^{3-7}$ The Lactobacillus species of gram-positive bacteria and the Bacillus spp. microbial strains are the primary micro-organisms used as probiotics. $^{8}$ Zhengchangsheng ${ }^{\circledR}$ (Bacillus Licheniformis) is a gram-positive, probiotic bacterial agent originally developed in China and approved for use in Korea in 1999.

This double-blind, randomized, multi-center study was conducted to assess the therapeutic efficacy and safety of Zhengchangsheng ${ }^{\circledR}$ capsules (Bacillus Licheniformis) as compared to Bioflor ${ }^{\circledR}$ capsules (Saccharomyces Boulardii) in patients with diarrhea caused by a wide range of conditions.

\section{METHODS}

\section{Subjects}

This study included patients between 20-75 years of age who had formless stools more than 3 times in 24 hours and more than 5 times in 48 hours from May 14, 2012-February 1,2013 . Patients with drug hypersensitivity to probiotic agents, lactose intolerance, IBD, diarrhea-predominant IBS, malabsorption, gastrointestinal bleeding, mechanical obstruction in the digestive tract, a history of antibiotic or antibacterial drug use within 1 week, and the use of anti-diarrheal or anti-spasmodic agents within 1 day were excluded. Pregnant or breastfeeding women were also excluded. Furthermore, investigators excluded patients with severe or uncontrollable endocrine or metabolic diseases, such as diabetes orthyroid diseases, severe or uncontrollable mental disorders, and severe internal and neurologic diseases. Patients currently under medication or who had participated in another clinical trial over the past 2 months were also excluded.

\section{Methods}

This study was performed in the Kyungpook National University Hospital, Keimyung University School of Medi- cine, Ewha Womans University Mokdong Hospital, Daegu Catholic University Medical Center, and Yeungnam University Hospital in accordance with Good Clinical Practice standards after gaining institutional review board approval from each institution. Patients with diarrhea who consented to participate in the trial were double-blinded, randomly assigned to treatment with either an investigational product (Zhengchangsheng ${ }^{\circledR}$ capsule [Bacillus Licheniformis $250 \mathrm{mg}$ ], Aju Pharm., Pyeongtaek, Gyeonggi, Korea) or a comparator (Bioflor ${ }^{\circledR}$ capsule [Saccharomyces Boulardii 250 mg], Kuhnil Pharm, Cheonan, Chungcheong, Korea), and then followed-up. The experimental group was administered 4 capsules ( 2 capsules Zhengchangsheng ${ }^{\circledR}+2$ Bioflor ${ }^{\circledR}$ placebo capsules), 3 times per day before or after meals. The comparison group was administered 4 capsules (twice a day $\left[\right.$ Bioflor $^{\circledR} 2$ capsules + Zhengchangsheng ${ }^{\circledR} 2$ placebo capsules], once a day [Bioflor ${ }^{\circledR} 2$ placebo capsules + Zhengchangsheng ${ }^{\circledR} 2$ placebo capsules]), 3 times per day before or after meals. Subjects were allowed to take medications prescribed before participating in this trial, but forbidden to take other probiotics, anti-diarrheal medicines, anti-spasmodic agents, antibiotics, antimicrobial agents, gastrointestinal stimulants, or anti-cholinergic drugs. The efficacy of the medications was evaluated for 5 days by reviewing the presence or absence of stool forms, changes in the number of daily bowel movements, improvements in subjective symptoms, and changes in the severity of diarrhea through self-administered surveys. Subjects were instructed to stop taking their study medications when their stools appeared in shape during the 5-days of medication use. Stools from hard to slightly spreading shapes were defined as formed feces. Stool shapes were evaluated based on the forms (types 1-4) in the Bristol Stool Form Scale (Table 1).

Based on a 0-3 scale, the survey assessed 5 items on subjective symptoms including abdominal pain, abdomi-

Table 1. Bristol Stool Form Scale

\begin{tabular}{lll}
\hline Type $^{*}$ & \multicolumn{1}{c}{ Form of human faeces } \\
\hline 1 & Separate hard lumps, like nuts (hard to pass) \\
3 & Sausage-shaped but lumpy \\
4 & Like a sausage but with cracks on its surface \\
5 & Sike a sausage or snake, smooth and soft \\
7 & Wluffy pieces with ragged edges, a mushy stool \\
\hline
\end{tabular}

*Formed feces were defined as type 1-4. 
nal distension, urgency for bowel movements, nausea, and vomiting. On the $0-3$ scale, absence of symptoms $=0$, mild symptoms/almost no previously unfelt suffering $=1$, moderate symptoms that did not interfere with daily activities $=2$, and severe symptoms that interfered with daily activities $=3$. The severity of diarrhea was assessed with a $100 \mathrm{~mm}$ visual analogue scale (VAS). Adverse events (AEs), prevalence, and vital signs were compared between the 2 groups to assess safety.

Investigators and clinical trial pharmacists instructed subjects to take each 1 of the 2 different medicines at a time. Subjects were provided with a medication teaching guide in order to take an accurate dosage, and asked to return any remaining medicine during their next visit. A conformity index (\%) was calculated with the amount of returned medicine as follows: (the amount of medicine taken divided by the total amount of medicine to be taken 3 times a day from drug administration start to end day) multiplied by 100 .

\section{Statistical Analysis}

This study was designed as a non-inferiority trial to assess whether the efficacy of the investigational product was not inferior to that of comparator. The equation for obtaining the number of required subjects is as follows:

$$
n=\frac{\left(z_{\alpha}+Z_{\beta}\right)^{2}\left(p_{c} q_{c}+p_{t} q_{t}\right)}{\left(\varepsilon-\left(p_{c}-p_{c}\right)^{2}\right.}
$$

Assuming that: (1) the ratio of sample sizes for the investigational product and comparator was $\lambda=1$; (2) an allowable error limit was $\varepsilon=0.2$, implying non-inferiority between the 2 groups; (3) predicted rates of symptom improvement were $P \mathrm{c}=0.85$ for the comparator and $P \mathrm{t}=0.85$ for the investigational product; (4) a significance level of $\alpha=0.05$; and (5) a type II error of $\beta=0.10$ (Power $=90 \%$ ), the minimum number of subjects required in each group was 67 . Considering a withdrawal rate of $15 \%$, the total number of subjects was 158 , requiring 79 participants in each group.

Data gathered were mainly divided into a full-analysisset (FAS), a per-protocol (PP) set, and a safety set. The FAS abided by the principles of an intention-to-treat population and included subjects with at least 1 measured efficacy variable among patients administered study medications at least once. The PP set comprised subjects that had completed the clinical trial according to the protocol, while the safety set consisted of subjects administered study medications after consenting to participate. Analysis of efficacy was performed in both the PP and FAS groups, and an analysis of safety was conducted in all subjects who received medications (safety group). Regarding data requiring before and after comparisons such as vital signs, completers according to the protocol (PP group) were analyzed.

\section{1) Analysis of Basic Demographic Data}

The data from all subjects were evaluated by therapeutic group. Mean, SD, and minimum and maximum values were calculated for continuous data, while frequencies and ratios were calculated for categorical data. Continuous variables were compared by conducting 2 sample $t$-tests or Wilcoxon rank-sum tests, and categorical variables were compared by conducting chi-square tests or Fisher's exact tests. Subgroup analyses were performed for variables showing a statistically significant difference.

\section{2) Evaluation and Analysis of Efficacy}

Investigators analyzed whether the lower bounds of a 95\% CI were greater than a non-inferiority margin of $-20 \%$ in efficacy rates (recovery to formed stools after study drug administration was deemed effective) on the 5th day after study drug administration between the experimental and control groups. The duration of diarrhea changing to formed feces after study drug administration was presented as the mean $\pm \mathrm{SD}$, and the difference between the 2 groups was compared through survival analysis. Moreover, continuous data such as changes in daily stool frequency as compared to Day 1 after study drug administration, the amount of improvement in subjective symptoms after study drug administration, and the difference in the $100 \mathrm{~mm}$ VAS score indicating the severity of diarrhea after study drug administration were presented as the mean $\pm \mathrm{SD}$, and analyzed by performing 2-sample $t$-tests or Wilcoxon rank-sum tests. Categorical variables such as the amount of improvement in subjective symptoms after study drug administration were presented as frequencies and ratios, and analyzed by conducting chi-square tests or Fisher's exact tests. In addition, subgroup analyses were performed on the evaluation results of efficacy variables according to the causes of diarrhea.

\section{3) Evaluation and Analysis of Safety}

All AEs were compiled from subjects with 1 or more AEs and the occurrence rates of AEs appearing before and after administration of study medications were calculated. Moreover, AEs appearing after administration of study medication were analyzed to determine which represented serious AEs, as well as the severity of AEs, the AEs that caused withdraw- 
al, and AEs associated with study medication, all of which were compared between the 2 groups. Paired $t$-tests were conducted to compare vital signs between the Bacillus and Saccharomyces groups. Unpaired $t$-tests or Wilcoxon ranksum tests were performed between the 2 groups.

\section{RESULTS}

\section{Characteristics of Subjects}

A total of 158 patients comprising the FAS participated in this trial and received either the investigational drug or the comparator. There were 78 and 80 subjects in the experimental and control groups, respectively. In the experimental group, 4 subjects dropped out ( 3 cases for violation of inclusion criteria, 1 case for failure to follow-up) and 3 subjects dropped out of the control group (3 cases for violation of inclusion criteria). This resulted in 74 and 77 subjects in the experimental and control groups, respectively, for the PP analysis (Fig. 1). There were no significant differences in gender, age, formless feces over 3 times during the 24 hours before screening, causes of diarrhea, history of medication use, or the presence of combined medication between the 2 groups (Table 2).

\section{Efficacy Evaluation}

\section{1) Rate of Recovery to Formed Feces}

When stool forms were examined from drug administration start to end day in the FAS analysis, the percentages of subjects with confirmed recovery of formed feces were 91.0\% (71/78) in the experimental (Zhengchangsheng ${ }^{\circledR}$ ) group and $95.0 \%(76 / 80)$ in the control (Bioflor ${ }^{\circledR}$ ) group. No statistically significant difference was found between the 2 groups $(P=0.326)$. In the PP analysis, the percentages of subjects with formed feces were $90.54 \%(67 / 74)$ in the experimental group and $96.10 \%(74 / 77)$ in the control group. No statistically significant difference was found between the 2 groups ( $P=0.169$; Fig. 2 ).

\section{2) Duration for Diarrhea Changing to Formed Feces}

A total of 147 subjects among the 158 subjects in the FAS group showed the recovery of formed feces after taking the investigational drug. According to the FAS analysis, the mean duration for recovery (diarrhea changing to formed feces) was $3.15 \pm 1.10$ days in the experimental (Zhengchangsheng $^{\circledR}$ ) group and $3.22 \pm 1.01$ days in the control (Bioflor ${ }^{\circledR}$ ) group. In the PP group, 141 of 151 subjects showed the recovery of formed feces. According to the PP analysis, the mean duration for recovery was $3.13 \pm 1.13$ days in the experimental (Zhengchangsheng ${ }^{\circledR}$ ) group and $3.20 \pm 1.01$ days in the control (Bioflor ${ }^{\circledR}$ ) group. Although the duration was shorter in the experimental group, no statistically significant difference was found between the 2 groups (FAS, $P=0.695$; PP, $P=0.704$ ). Kaplan-Meier survival analysis was performed on the duration for recovery to formed feces in the FAS and PP groups. According to the results, the graphed survival curves were comparable for the 2 groups. More than $50 \%$ of patients with diarrhea were recovered on the 3rd day, and almost all patients were fully recovered on the 5 th day after

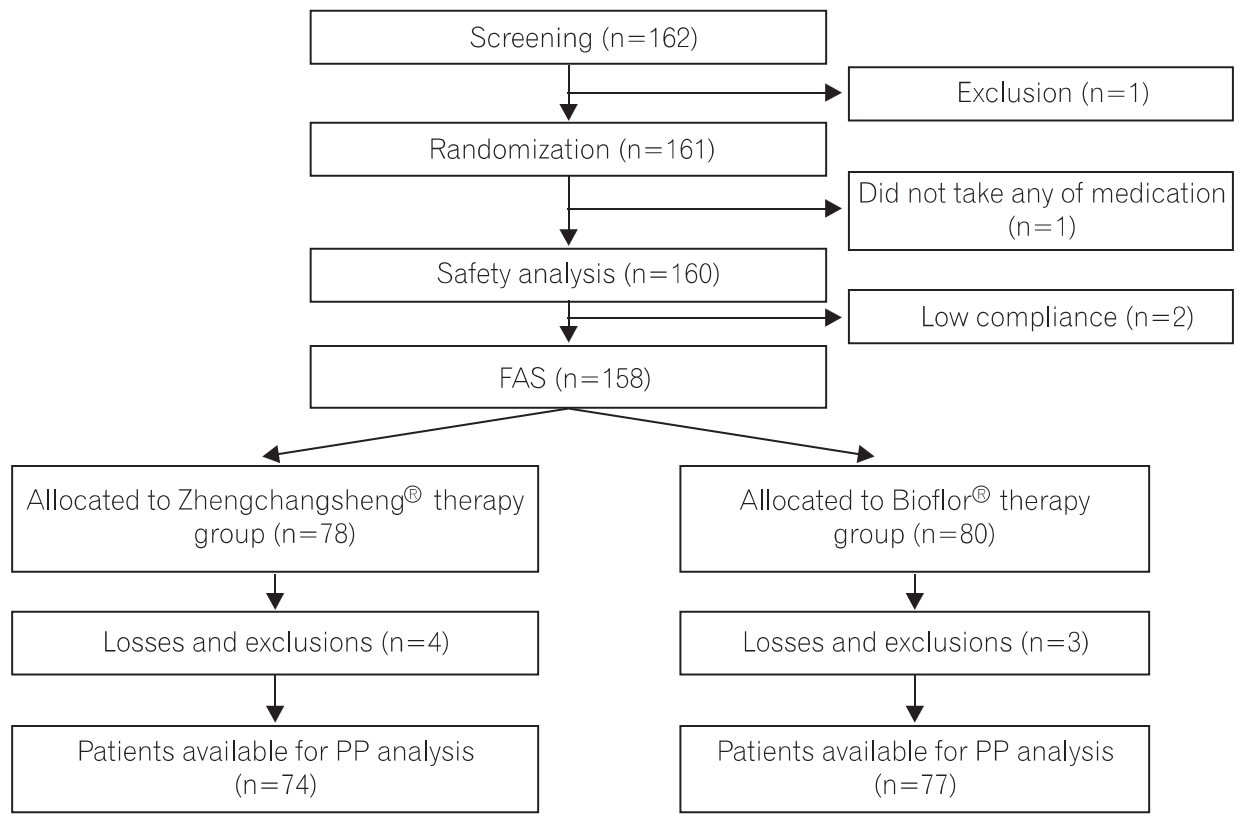

Fig. 1. Flow diagram showing entries and withdrawals from the study. FAS, full-analysis-set; PP, per-protocol. 
Table 2. Baseline Characteristics of Patients (full-analysis-set)

\begin{tabular}{|c|c|c|c|c|c|}
\hline Characteristics & & Zhengchangsheng $^{\circledR}(n=78)$ & Bioflor $^{\circledR}(n=80)$ & Total $(n=158)$ & $P$-value \\
\hline \multirow[t]{2}{*}{ Sex } & Male & $39(50.0)$ & $37(46.3)$ & $76(48.1)$ & 0.637 \\
\hline & Female & $39(50.0)$ & $43(53.7)$ & $82(51.9)$ & \\
\hline Age (yr) & Mean $\pm S D$ & $36.8 \pm 11.7$ & $36.8 \pm 10.7$ & $36.8 \pm 11.2$ & 0.986 \\
\hline \multirow[t]{2}{*}{ Formless feces over 3 times for 24 hours } & Existence & 71 (91.0) & $70(87.5)$ & 141 (89.2) & 0.475 \\
\hline & None & $7(8.97)$ & $10(12.5)$ & $17(10.8)$ & \\
\hline \multirow[t]{4}{*}{ Causes of diarrhea } & Viral diarrhea & $14(18.0)$ & $14(17.5)$ & $28(17.7)$ & 0.261 \\
\hline & Bacterial diarrhea & 0 & $1(1.3)$ & $1(0.6)$ & \\
\hline & Functional diarrhea & 0 & 3 (3.8) & $3(1.9)$ & \\
\hline & Unknown & $64(82.1)$ & $62(77.5)$ & $126(79.8)$ & \\
\hline \multirow[t]{2}{*}{ Previous medication } & Existence & $2(2.6)$ & $6(7.5)$ & $8(5.1)$ & 0.157 \\
\hline & None & 76 (97.4) & $74(92.5)$ & $150(94.9)$ & \\
\hline \multirow[t]{2}{*}{ Combined medication } & Existence & $6(7.7)$ & $10(12.5)$ & 16 (10.1) & 0.317 \\
\hline & None & 72 (92.3) & 70 (87.5) & 142 (89.9) & \\
\hline
\end{tabular}

Values are presented as $n(\%)$.

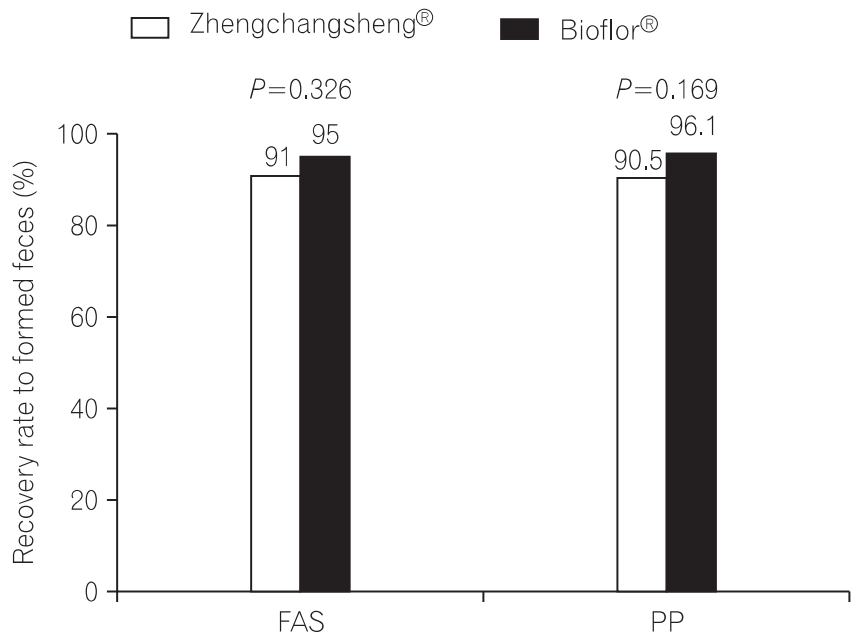

Fig. 2. Recovery rate to formed feces among patients treated with Zhengchangsheng ${ }^{\circledR}$ and Bioflor ${ }^{\circledR}$ for 5 days. FAS, full-analysis-set; PP, per-protocol.

receiving the investigational drug (Fig. 3).

\section{3) Changes in Daily Stool Frequency}

Changes in daily stool frequency compared to Day 1 of the administration of the investigational drug were examined using subject diaries. Consequently, a consistent decrease was identified in daily stool frequency from Day 1-Day 7. According to the FAS analysis, the change in daily stool frequency on the day that diarrhea changed to formed feces compared to Day 1 was $1.66 \pm 2.20$ times/day in the experimental group and $1.56 \pm 1.77$ times/day in the control group.
A statistically significant difference was detected in both groups (both $P<0.001$ ), but no statistically significant difference was detected between the 2 groups $(P=0.744)$. In the PP analysis, the experimental and control groups showed a change in daily stool frequency of $1.79 \pm 2.19$ and $1.63 \pm 1.75$ times/day, respectively. A statistically significant difference was detected in both groups (both $P<0.001$ ), however, no statistically significant difference was evident between the 2 groups $(P=0.638)$.

\section{4) Improvements in Subjective Symptoms}

Investigators examined improvements in 5 subjective symptoms including abdominal pain, abdominal distension, urgency for bowel movements, nausea, and vomiting by comparing Day 1 with Day 6. Statistically significant differences indicative of improvements in the 5 subjective symptoms were found for both treatment groups in the FAS analysis, however, no statistically significant differences were detected between the 2 groups (Fig. 4).

\section{5) Difference in the $\mathbf{1 0 0} \mathrm{mm}$ VAS Score Indicative of Severity of Diarrhea}

The difference in the $100 \mathrm{~mm}$ VAS used to evaluate the severity of diarrhea was $46.05 \pm 24.96 \mathrm{~mm}$ in the experimental group and $53.05 \pm 18.02 \mathrm{~mm}$ in the control group according to the PP analysis. The severity of diarrhea improved in both groups and a statistically significant difference was observed in the difference in the $100 \mathrm{~mm}$ VAS score in each group $(P<0.001)$. However, no statistically significant difference was 

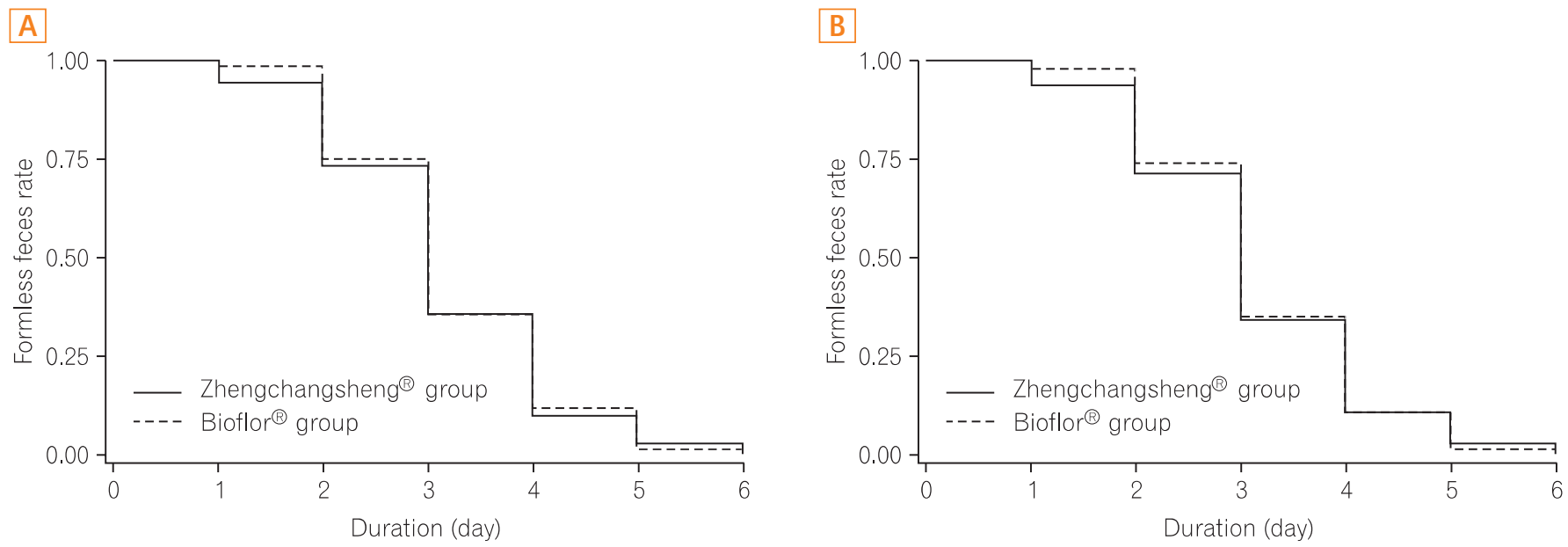

Fig. 3. Duration of recovery to formed feces after medication (Kaplan-Meier analysis). (A) Full-analysis-set. (B) Per-protocol analysis.

A

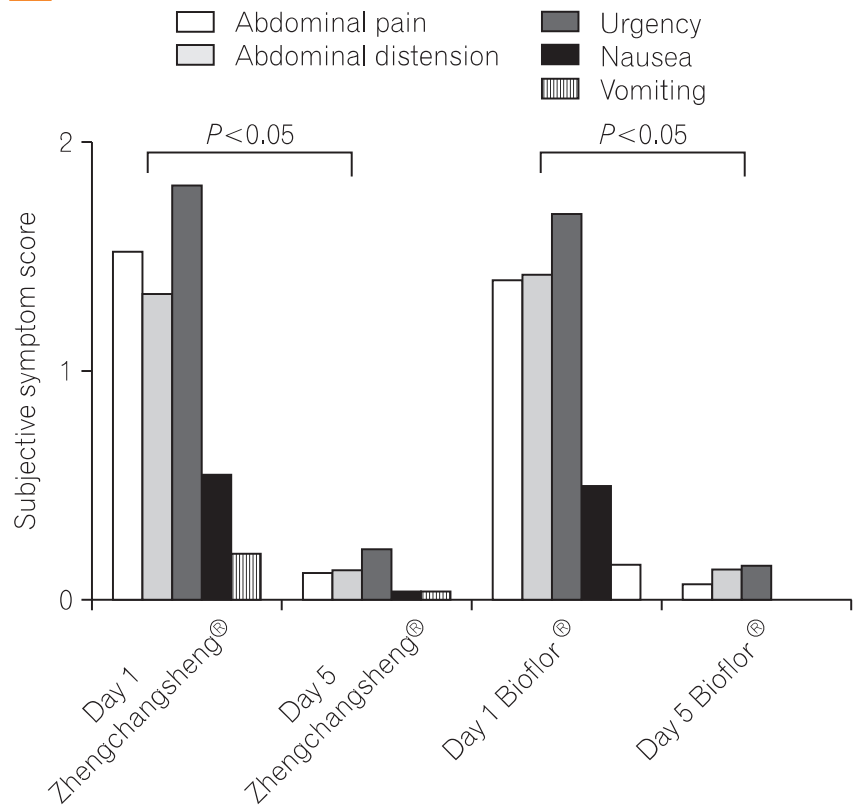

B
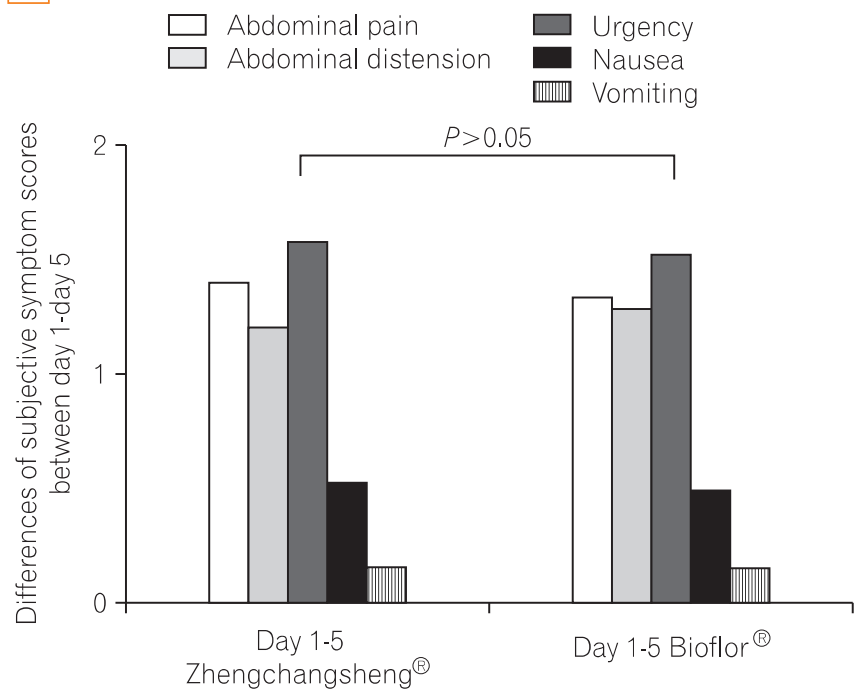

Fig. 4. Subjective symptom score among patients treated with Zhengchangsheng ${ }^{\circledR}$ and Bioflor ${ }^{\circledR}$ for 5 days. (A) Subjective symptom score on day 1 and day 5. (B) Differences in subjective symptom scores between day 1-day 5.

detected between the 2 groups $(P=0.159)$.

\section{6) Conformity Index}

In the FAS analysis, conformity indices were $101.9 \%$ in the experimental group and $95.7 \%$ in the control group, and showed no significant difference between the 2 groups.

\section{Non-Inferiority Test}

In the non-inferiority test for the rate of recovery to formed feces, the lower bounds of a $95 \%$ CI were $-11.9 \%$ in the FAS analysis and $-13.5 \%$ in the PP analysis, greater than a non-inferiority margin of $-20 \%$ (Table 3 ). Therefore, non-inferiority was confirmed in the experimental group as compared to the control group.

\section{Adverse Events}

An AE occurred in 1 of the 159 subjects during this clinical trial, and the occurrence rate was $1.28 \%$. No statistically significant differences were detected in the occurrence rates between the 2 groups $(P=0.491)$. There were no AEs 
Table 3. Analysis of Non-inferiority in the Full-Analysis-set (FAS) and Per-Protocol (PP) Populations

\begin{tabular}{llrrr}
\hline & & Zhengchangsheng $^{\circledR}$ & Bioflor $^{\circledR}$ & Zhengchangsheng $^{\circledR}$ - Bioflor \\
\hline FAS analysis & The rate of recovery to formed feces, $\%(95 \% \mathrm{Cl})$ & $91.0(94.68-97.37)$ & $95.0(90.22-99.78)$ & $(-11.9-3.97)$ \\
PP analysis & The rate of recovery to formed feces, \% (95\% Cl) & $90.5(83.87-97.21)$ & $96.1(91.78-100.0)$ & $(-13.5-2.38)$ \\
\hline
\end{tabular}

or serious AEs considered to be associated with the study drugs. The single observed AE was mild constipation which recovered completely without any after effects. This AE was considered to be unrelated to the investigational drug. AErelated care or treatment measures were not taken. No statistically significant differences between the 2 groups were observed in vital signs measured after taking the study medications.

\section{DISCUSSION}

Bioflor ${ }^{\circledR}$ (Saccharomyces boulardii) is a widely used medication that has demonstrated the ability to improve the duration and symptoms of acute diarrhea without specific adverse reactions in several studies. ${ }^{9-11}$ This study was a double-blind, randomized, multi-center clinical trial conducted to identify the effects of treatment with Zhengchangsheng ${ }^{\circledR}$ capsules on patients with diarrhea attributable to various causes via comparison with a control group treated with Bioflor ${ }^{\circledR}$ capsules. The study results revealed that Zhengchangsheng $^{\circledR}$ is not inferior to Bioflor ${ }^{\circledR}$. Moreover, clinically significant AEs were not observed in either groups, and the efficacy and safety of both treatments were confirmed in patients with diarrhea attributable to various factors.

Various effective and beneficial probiotic micro-organisms have been used to prevent and manage specific pathologic conditions. In theory, the benefits attributable to probioticsare due to the protective effects of the normal intestinal flora against disease. The normal intestinal flora have been shown to play an important role in preventing invasion by pathogens in several in vivo and in vitro tests. ${ }^{12}$ Probiotics play positive roles in maintaining and recovering the normal intestinal flora. First, probiotics produce $\mathrm{H}_{2} \mathrm{O}_{2}$, organic acid, bacteriocin, and other defensive compounds as part of a protective mechanism. Second, they protect the intestine by competing with pathogens in the intestinal mucosal barrier for attachment. ${ }^{13,14}$ Probiotics also compete with pathogenic bacteria for nutrients in the gut. Additionally, they may degrade toxin receptors. ${ }^{15-17}$ Furthermore, probiotics can stimulate protective mechanisms against bowel diseases via interaction with the immune system. ${ }^{18-20}$

The most commonly used probiotics are Lactobacil- lus spp. And Bacillus spp. Bacillus licheniformis is a grampositive, thermophilic, and spore-forming bacterium commonly found in the soil. It has been used in the production of other chemical substances including enzymes and antibiotics through fermentation processes, and is generally considered as a non-pathogen. ${ }^{8,21,22}$ Zhengchangsheng ${ }^{\circledR}$ (Bacillus Licheniformis) is a safe, human-derived probiotic initially developed in China, and was approved by Korea's regulatory authorityin 1999. Unlike other probiotic agents, Zhengchangsheng ${ }^{\circledR}$ inhibits the growth of harmful pathogens without affecting the $\mathrm{pH}$ of the intestine. ${ }^{23}$ According to the results of in vitro tests performed in Korea using Bacillus licheniformis identified in fermented soybeans (chungookjang), antimicrobial activities were observed in Escherichia coli, Staphylococcus aureus, and Candida albicans. ${ }^{24}$ Another domestic study demonstrated the antimicrobial activity of Zhengchangsheng ${ }^{\circledR}$ in major pathogens associated with infectious diarrhea including Escherichia coli, Salmonella typhi, Shigella sonnei, and others. Moreover, an infection inhibition effect was seen in Rotaviru. ${ }^{23}$ According to a recent study from Newcastle University, UK, Bacillus Licheniformis demonstrated the ability to disperse biofilms in both grampositive and gram-negative bacteria, and extracellular DNase (NucB) was proven to take this role. ${ }^{25}$ Additional studies will be necessary in order to further elucidate the mechanisms underlying the of antimicrobial activity of Bacillus licheniformis towards harmful bacteria.

Antibiotics are used to treat a wide range of infections and have a significant impact on the intestinal flora. Imbalanced intestinal flora generated by the use of antibiotics provokes abnormal gut fermentation, and eventually causes clinical symptoms including diarrhea. Antibiotic-related diarrhea is directly associated with Clostridium difficile infections. The prevalence of those diseases is increasing in concert with recent increases in the use of antibiotics. In a recent study that analyzed 25 randomized comparative studies on the efficacy of probiotics for the prevention of antibiotic-related diarrhea, a meaningful decrease in antibiotic-related diarrhea was detected (relative risk $=0.43,95 \% \mathrm{CI}=0.31-0.58, P<0.001$ ). Moreover, 6 randomized comparative studies also showed the effectiveness of probiotics in Clostridium difficile-associated diseases (relative risk $=0.59,95 \% \mathrm{CI}=0.41-0.85, P=0.005){ }^{26}$ 
In a previous study of the effectiveness of probiotics in 50 patients with diarrhea-predominant IBS, a significant improvement was observed in the experimental group as compared to the placebo control group ( $48 \%$ vs. $12 \%, P=0.01$ ), and stool density also significantly improved ${ }^{27}$ Furthermore, a comparative study of 129 patients with IBS-associated functional dyspepsia conducted in patients treated with either trimebutine or Zhengchangsheng ${ }^{\circledR}$ alone or in combination showed significant improvements and comparable results for all 3 groups. ${ }^{28}$ However, the results from a randomized, double-blind, placebo-controlled comparative study on the effects of probiotics showed insignificant symptom improvement in patients with IBS. ${ }^{29,30}$ Collectively, these early studies suffered from methodological problems including small sample sizes and poor patient compliance. Therefore, large-scale studies will be required to further investigate the usefulness of probiotics in patients with IBS.

There were some limitations in this study. Because this trial comprised subjects with the clinical manifestations of diarrhea, the results were somewhat inconsistent. Moreover, the causes of acute diarrhea varied, as subjects consisted of both acute and chronic diarrhea patients. To more accurately verify the treatment effects of probiotics in patients with diarrhea, additional studies will be required to distinguish acute diarrhea from chronic diarrhea, or infectious diarrhea from non-infectious diarrhea.

In conclusion, Zhengchangsheng ${ }^{\circledR}$ is a probiotic agent that has been proven to be clinically effective in treating abnormal gut fermentation and diarrhea attributable to various causes through both the inhibition of the growth of pathogens associated with diarrhea and the normalization of intestinal flora functioning. This study was able to verify that Zhengchangsheng ${ }^{\circledR}$ was not inferior to Bioflor ${ }^{\circledR}$ in therapeutic efficacy and was a safe and useful therapeutic agent for the treatment of diarrhea. Therefore, Zhengchangsheng ${ }^{\circledR}$ is expected to be not only clinically effective, but also attractive as a therapeutic option due to its exceedingly mild tolerability profile.

\section{REFERENCES}

1. de Vrese M, Marteau PR. Probiotics and prebiotics: effects on diarrhea. J Nutr 2007;137:803S-811S

2. Eizaguirre I, Urkia NG, Asensio AB, et al. Probiotic supplementation reduces the risk of bacterial translocation in experimental short bowel syndrome. J Pediatr Surg 2002;37:699-702.

3. Billoo AG, Memon MA, Khaskheli SA, et al. Role of a probiotic (Saccharomyces boulardii) in management and prevention of diarrhoea. World J Gastroenterol 2006;12:4557-4560.

4. Whorwell PJ, Altringer L, Morel J, et al. Efficacy of an encapsulated probiotic Bifidobacterium infantis 35624 in women with irritable bowel syndrome. Am J Gastroenterol 2006;101:15811590.

5. Oberhelman RA, Gilman RH, Sheen P, et al. A placebo-controlled trial of Lactobacillus GG to prevent diarrhea in undernourished Peruvian children. J Pediatr 1999;134:15-20.

6. Dendukuri N, Costa V, McGregor M, Brophy JM. Probiotic therapy for the prevention and treatment of Clostridium difficileassociated diarrhea: a systematic review. CMAJ 2005;173:167170.

7. McFarland LV. Meta-analysis of probiotics for the prevention of traveler's diarrhea. Travel Med Infect Dis 2007;5:97-105.

8. Rolfe RD. The role of probiotic cultures in the control of gastrointestinal health. J Nutr 2000;130:396S-402S.

9. Gaon D, Garcia H, Winter L, et al. Effect of Lactobacillus strains and Saccharomyces boulardii on persistent diarrhea in children. Medicina (B Aires) 2003;63:293-298.

10. Bleichner G, Blehaut H, Mentec H, Moyse D. Saccharomyces boulardii prevents diarrhea in critically ill tube-fed patients. A multicenter, randomized, double-blind placebo-controlled trial. Intensive Care Med 1997;23:517-523.

11. Htwe K, Yee KS, Tin M, Vandenplas Y. Effect of Saccharomyces boulardii in the treatment of acute watery diarrhea in Myanmar children: a randomized controlled study. Am J Trop Med Hyg 2008;78:214-216.

12. Fuller R. Probiotics in human medicine. Gut 1991;32:439-442.

13. Conway PL, Gorbach SL, Goldin BR. Survival of lactic acid bacteria in the human stomach and adhesion to intestinal cells. J Dairy Sci 1987;70:1-12

14. Goldin BR, Gorbach SL, Saxelin M, Barakat S, Gualtieri L, Salminen S. Survival of Lactobacillus species (strain GG) in human gastrointestinal tract. Dig Dis Sci 1992;37:121-128.

15. Castagliuolo I, LaMont JT, Nikulasson ST, Pothoulakis C. Saccharomyces boulardii protease inhibits Clostridium difficile toxin A effects in the rat ileum. Infect Immun 1996;64:52255232.

16. Castagliuolo I, Riegler MF, Valenick L, LaMont JT, Pothoulakis C. Saccharomyces boulardii protease inhibits the effects of Clostridium difficile toxins A and B in human colonic mucosa. Infect Immun 1999;67:302-307.

17. Pothoulakis C, Kelly CP, Joshi MA, et al. Saccharomyces boulardii inhibits Clostridium difficile toxin A binding and enterotoxicity in rat ileum. Gastroenterology 1993;104:1108-1115.

18. Fukushima Y, Kawata Y, Hara H, Terada A, Mitsuoka T. Effect of a probiotic formula on intestinal immunoglobulin A production in healthy children. Int J Food Microbiol 1998;42:39-44. 
19. Malin M, Suomalainen H, Saxelin M, Isolauri E. Promotion of IgA immune response in patients with Crohn's disease by oral bacteriotherapy with Lactobacillus GG. Ann Nutr Metab 1996;40:137-145.

20. Kaila M, Isolauri E, Soppi E, Virtanen E, Laine S, Arvilommi H. Enhancement of the circulating antibody secreting cell response in human diarrhea by a human Lactobacillus strain. Pediatr Res 1992;32:141-144.

21. de Boer AS, Priest F, Diderichsen B. On the industrial use of Bacillus licheniformis: a review. Appl Microbiol Biotechnol 1994; 40:595-598.

22. Rowland I. Probiotics and benefits to human health--the evidence in favour. Environ Microbiol 1999;1:375-376.

23. Kim JY, Bae EA, Han MJ, Kim DH. Inhibitory activity of Bacillus licheniformis $\mathrm{AJ}$ on the growth of diarrheal pathogens. Biomol Ther 1999;7:385-389.

24. Kim Y, Cho JY, Kuk JH, et al. Identification and antimicrobial activity of phenylacetic acid produced by Bacillus licheniformis isolated from fermented soybean, Chungkook-Jang. Curr Microbiol 2004;48:312-317.
25. Nijland R, Hall MJ, Burgess JG. Dispersal of biofilms by secreted, matrix degrading, bacterial DNase. PLoS One 2010;5:e15668.

26. McFarland LV. Meta-analysis of probiotics for the prevention of antibiotic associated diarrhea and the treatment of Clostridium difficile disease. Am J Gastroenterol 2006;101:812-822.

27. Ki Cha B, Mun Jung S, Hwan Choi C, et al. The effect of a multispecies probiotic mixture on the symptoms and fecal microbiota in diarrhea-dominant irritable bowel syndrome: a randomized, double-blind, placebo-controlled trial. J Clin Gastroenterol 2012;46:220-227.

28. Zhong YQ, Zhu J, Guo JN, et al. A randomized and case-control clinical study on trimebutine maleate in treating functional dyspepsia coexisting with diarrhea-dominant irritable bowel syndrome. Zhonghua Nei Ke Za Zhi 2007;46:899-902.

29. O'Sullivan MA, O'Morain CA. Bacterial supplementation in the irritable bowel syndrome. A randomised double-blind placebocontrolled crossover study. Dig Liver Dis 2000;32:294-301.

30. Kim YG, Moon JT, Lee KM, Chon NR, Park H. The effects of probiotics on symptoms of irritable bowel syndrome. Korean J Gastroenterol 2006;47:413-419. 